УДК 621.771

\author{
Е.И. Шифрин ${ }^{1}$, В.Ф. Балакин ${ }^{1}$, Н.Ю. Квитка ${ }^{1,2}$ \\ ${ }^{1}$ Национальная металлургическая академия Украины \\ ${ }^{2}$ George Brown College, Toronto, ON, Canada
}

\title{
АППРОКСИМИРУЮЩЕЕ УРАВНЕНИЕ ДЛЯ ОПРЕДЕЛЕНИЯ КОЭФФИЦИЕНТА ПОДПОРА ПРИ ПРОДОЛЬНОЙ ПРОКАТКЕ ПОЛОСЫ
}

\begin{abstract}
Расчет коэффициента подпора при прокатке - процедура сложная и требующая значительных затрат времени как при составлении вычислительных программ, так и при их реализации. Для упрощения и ускорения вычислительных процедур предложено уравнение, аппроксимирующее значения коэффициента подпора $n_{\text {би }}$, pacсчитанные при помощи модифицированного метода баланса работ. Ошибка аппроксимации расчётных данных не превышает $-12 \ldots+15 \%$, что вполне приемлемо для инженерных расчётов. Применение соответствующих критериев-аналогов и разработанного аппроксимирующего уравнения, позволило выполнить расчёты силовых параметров деформации в станах трубопрокатных агрегатов "30-102", "350", "140" и "80".
\end{abstract}

DOI: $10.34185 / 0543-5749.2020-2-3-9$

\section{Введение}

В 1939 году А.И.Целиков вывел известную формулу для расчёта коэффициента подпора $n_{\sigma}$ при прокатке полос в гладких валках [1]. Для упрощения ведения расчётов в 1940 году М.А .Лакс построил по этой формуле графики, которые в специальной литературе известны как "графики Целикова" [2]. По этим графикам величину $n_{\sigma}$ определяют как функцию показателей $\delta=\frac{2 f l_{d}}{\Delta h}$ (здесь $f-$ коэффициент внешнего трения; $l_{d}$ - длина очага деформации; $\Delta h-$ изменение высоты полосы в процессе прокатки) и $\varepsilon=\frac{\Delta h}{h_{0}}$ (здесь $h_{0}-$ высота полосы до деформации). Такая методика определения коэффициента подпора $n_{\sigma}$ применима в случае прокатки тонких полос, когда выполняется условие $1<\frac{l_{d}}{h_{c p}}$ (здесь $h_{c p}=h_{0}-\frac{\Delta h}{2}-$ средняя высота полосы). При прокатке высоких полос, когда $1>\frac{l_{d}}{h_{c p}}$, А.И. Целиков и А.И. Гришков рекомендуют определять $n_{\sigma}$ по формуле, предложенной в работе [3, стр. 104].

В работе [4] предложен базирующийся на модифицированном методе баланса работ принцип расчёта коэффициентов подпора при простой продольной прокатке полосы. Рассчитанные по этому методу значения коэффициента подпора $n_{\text {би }}$, в отличие от коэффициентов подпора $n_{\sigma}$, могут быть использованы при расчёте силовых параметров прокатки во всём диапазоне возможных значений показателя $\frac{l_{d}}{h_{c p}}$.

Следует отметить, что, даже при использовании современных автоматизированных систем математических вычислений, расчёт коэффициентов подпора $n_{\sigma}$ и $n_{\sigma u}-$ процедура сложная и требующая значительных затрат времени как при составлении вычислительных программ, так и при их реализации. Поэтому в инженерных методиках при расчёте коэффициента

(с Шифрин Е.И., Балакин В.Ф., Квитка Н.Ю., 2020 
подпора $n_{\sigma}$ часто используют не методику А.И. Целикова, а аппроксимирующие уравнения, например [5, стр. 115], следующего вида

$$
n_{\sigma}^{\prime}=1+\frac{f l_{d}}{h_{c p}}
$$

Зависимость (1) аппроксимирует значения коэффициентов подпора, рассчитанные по уравнению А.И. Целикова, с ошибкой $\Delta=\frac{n_{\sigma}-n_{\sigma}^{\prime}}{n_{\sigma}} \cdot 100 \%, \quad$ не $\quad$ превышающей $20 \ldots+10 \%$ для реальных значений $\varepsilon$ и $f$ (рис. 1).

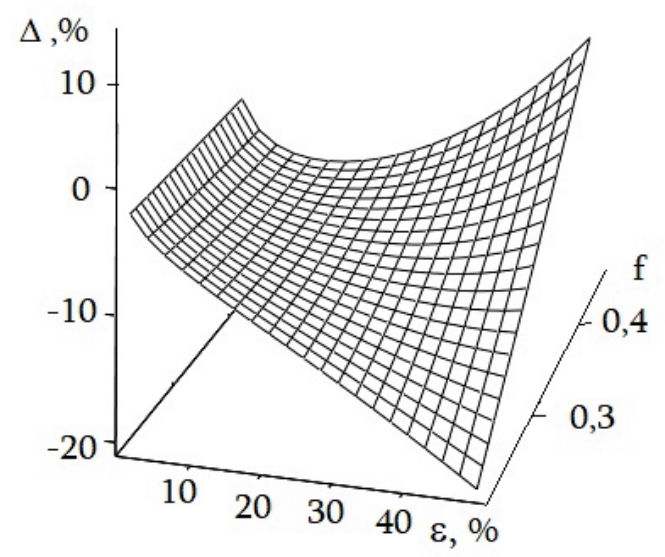

Рис. 1. Ошибка аппроксимации $\Delta=\Delta(\varepsilon, f)$ уравнения А.А. Целикова зависимостью (1)

\section{Цель работы}

Предложить уравнение, аппроксимирующее значения коэффициента подпора $n_{\sigma u}$ с достаточной для инженерных расчётов точностью и, тем самым, упростить определение силовых параметров технологических процессов прокатки.

\section{Постановка задачи}

Рассчитанные по предложенному в работе [4] методу коэффициенты подпора $n_{\sigma u}=n_{\sigma u}(f, \varepsilon, \bar{R})$ есть функции от коэффициента трения $f$, относительного обжатия полосы $\varepsilon$ и относительного радиуса валка $\bar{R}=\frac{R}{h_{0}}$ (здесь $R$ - радиус валка). Необходимо получить зависимость $\quad n_{\sigma u}^{\prime}=n_{\sigma u}^{\prime}(f, \varepsilon, \bar{R}), \quad$ аппроксими- рующую расчётные значения $n_{\sigma u}=n_{\sigma u}(f, \varepsilon, \bar{R})$.

\section{Решение задачи}

В работе [6] предложен алгоритм статистической обработки эмпирических данных, который вполне применим к созданию уравнений, аппроксимирующих расчетные данные [7]. По этому алгоритму последовательность создания аппроксимирующего уравнения такова:

- выбирается базовое уравнение, характер изменения которого при изменении варьируемых параметров качественно соответствует характеру изменения расчётной зависимости;

- базовое уравнение дополняется функциями варьируемых параметров (так называемыми брик-функциями), целесообразность использования которых в аппроксимирующем уравнении обосновывается на основании сопоставления исходных расчётных данных и результатов расчёта по аппроксимирующей зависимости.

Учитывая, что качественно значения функции $n_{\sigma u}=n_{\sigma u}(f, \varepsilon, \bar{R})$ изменяются так же, как значения $n_{\sigma}$, рассчитанные по методикам, предложенным в работах $[1,3]$, в качестве базового уравнения использовали следующее логическое условие

$$
n_{\sigma u}^{\prime}=\mid \begin{aligned}
& 1+\frac{f l_{d}}{h_{c p}} \text { если } \frac{l_{d}}{h_{c p}} \geq 1 \\
& 1,25 \ln \frac{h_{c p}}{l_{d}}+0,25 \frac{l_{d}}{h_{c p}}-0,25 \text { если } \frac{l_{d}}{h_{c p}}<1 .
\end{aligned}
$$

Учитывая, что

$$
\frac{l_{d}}{h_{c p}}=\bar{L}=\frac{2 \bar{R}}{2-\varepsilon} \cdot \sqrt{\frac{\varepsilon}{2 \bar{R}}\left(2-\frac{\varepsilon}{2 \bar{R}}\right)}
$$

[8, стр. 38], базовое уравнение (2) является функцией тех же варьируемых параметров $f$, $\varepsilon$ и $\bar{R}$, что и исходная расчётная зависимость. Путём добавления к базовому уравнению (2) различных брик - функций, проверки целесообразности их добавления и соответствующих последовательных преобразований уравнение (2) принимает окончательный вид:

$$
n_{\sigma и}^{\prime}=G_{o} \cdot \mid \begin{aligned}
& (1+f)+(\bar{L}-1) k \text { если } \bar{L} \geq 1 \\
& \times(1+f \bar{L}) \text { если } \bar{L}<1 ;
\end{aligned}
$$




$$
n_{\sigma u}^{\prime}=\mid \begin{array}{lll}
n_{\sigma u}^{\prime} & \text { если } & n_{\sigma u}^{\prime}<2,856 \\
2,856 & \text { если } & n_{\sigma u}^{\prime} \geq 2,856,
\end{array}
$$

где $G_{o}=G_{o}(f, \varepsilon, \bar{R})$ - функция (методика определения приведена ниже);

$$
k=k(f, \bar{R})=k_{1} \ln \frac{\bar{R}}{10}+k_{2} ;
$$

$k_{1}=k_{1}(f)=-249,87 f^{3}+204 f^{2}-54,18 f+4,75$;

$k_{2}=k_{2}(f)=-2,65 f^{2}+2,17 f-0,39$.

Функция $G_{o}(f, \varepsilon, \bar{R})$ определяется следующим образом:

$$
G_{o}=\mid \begin{aligned}
& 1+\frac{0,1\left(\varepsilon-\varepsilon_{1}\right)}{\varepsilon_{2}-\varepsilon_{1}} \text { если } \varepsilon<\varepsilon_{1} \\
& 0,04 \ln \bar{R}+0,82 \text { если } \varepsilon \geq \varepsilon_{1}
\end{aligned},
$$

где $\varepsilon_{1}=\varepsilon_{1}(f, \varepsilon, \bar{R})-$ корень уравнения $\frac{n_{\sigma u}(f, \varepsilon, \bar{R})}{n_{\sigma u}^{\prime \prime}(f, \varepsilon, \bar{R})}=1,0$; $\varepsilon_{2}=\varepsilon_{2}(f, \varepsilon, \bar{R})$ - корень уравнения $\frac{n_{\sigma u}(f, \varepsilon, \bar{R})}{n_{\sigma u}^{\prime \prime}(f, \varepsilon, \bar{R})}=1,1$ $n_{\sigma u}^{\prime \prime}(f, \varepsilon, \bar{R})=\left[\Psi \ln \frac{1}{\bar{L}}+\Psi \bar{L}-(\Psi-1)\right] \times$. $\times(1+f \bar{L})(0,82+0,04 \ln \bar{R})$.

На рис. 2 приведены исходные значения коэффициентов подпора $n_{\text {би }}$, рассчитанные по методике [4], и значения $n_{\sigma u}^{\prime}$, рассчитанные по аппроксимирующему уравнению (2).
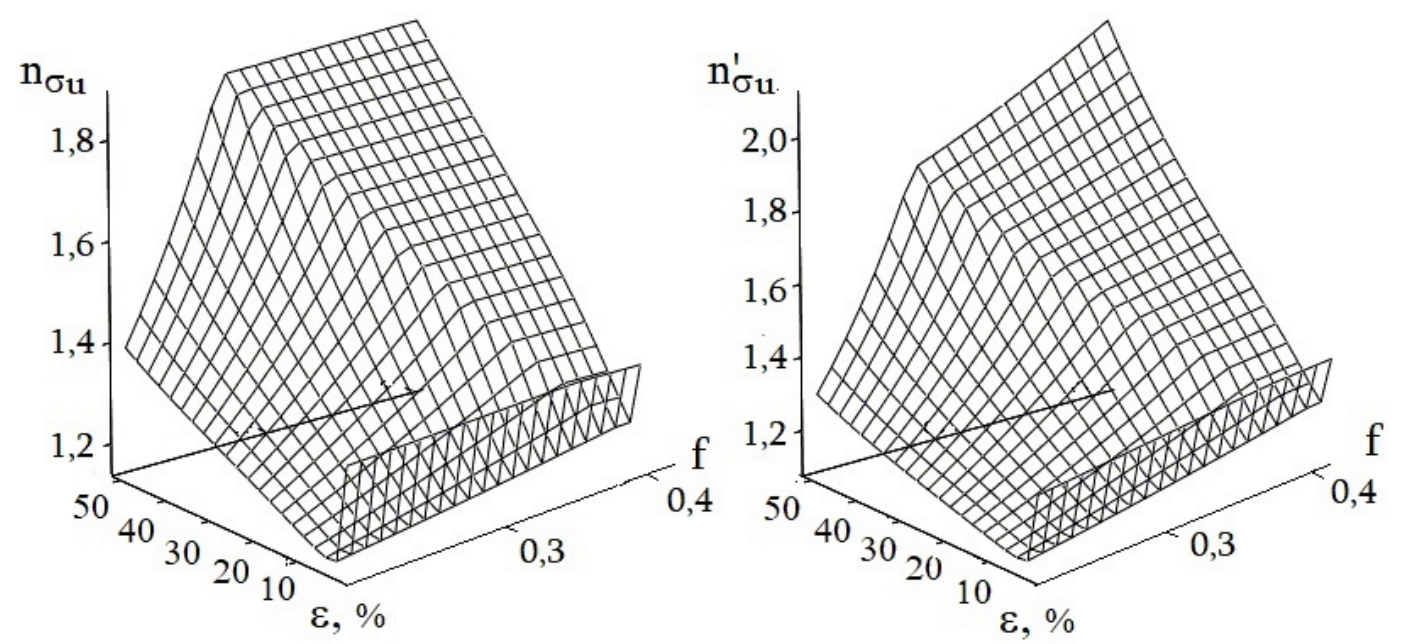

Рис. 2. Зависимости $n_{\sigma u}(f, \varepsilon, \bar{R}=25)$ и $n_{\sigma u}^{\prime}(f, \varepsilon, \bar{R}=25)$

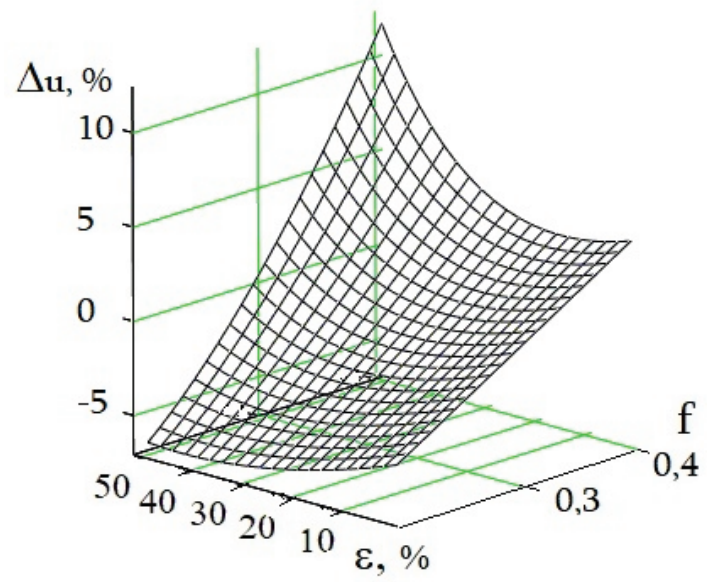

Pис. 3. Функция ошибки аппроксимации $\Delta_{u}(f, \varepsilon, \bar{R}=25)$ 
Анализ расчётных данных, представленных на рис. 2, позволяет сделать вывод о том, что тенденции изменения исходной $\left(n_{\sigma u}\right)$ и аппроксимирующей функций $\left(n_{\sigma u}^{\prime}\right)$ аналогичны.

На рис. 3 представлена зависимость ошибки аппроксимации $\Delta_{u}=\frac{n_{\sigma u}-n_{\sigma u}^{\prime}}{n_{\sigma u}} \cdot 100 \%$ от относительного обжатия $\varepsilon$ и коэффициента трения $f$ при $\bar{R}=25$.

Как следует из приведенных на рис. 3 данных ошибка аппроксимации расчётных значений коэффициента подпора $n_{\sigma и}$ лежит в пределах $\Delta_{u}=-7 \ldots+12 \%$. При варьировании величины $\bar{R}$ в пределах $0,25 \ldots 100$ ошибка $\Delta_{u}$ не выходит за рамки диапазона $-12 \ldots+15 \%$ что вполне приемлемо для инженерных расчётов.

\section{Практическое использование результатов}

Начиная с 1946 года и до настоящего времени формулу Целикова применяют в методиках расчётов силовых параметров деформации труб при поперечно-винтовой [9, стр. 457] и продольной прокатке [5, стр. 114]. В работе [7] показана возможность применения расчётных коэффициентов подпора $n_{\sigma u}$, полученных в работах [4, $10]$, при изучении процесса безоправочной прокатки труб. При использовании методик $[5,7,9$ и др.] геометрические параметры, характеризующие процесс продольной прокатки полосы, заменяют критериями-аналогами, характеризующими условия протекания конкретного процесса изготовления труб. Применение соответствующих критериев-аналогов и разработанного аппроксимирующего уравнения (3) позволило выполнить расчёты силовых параметров деформации в станах трубопрокатных агрегатов "30102", "350", "140" и "80". Эти данные были использованы при разработке таблиц прокатки и проектировании нового оборудования.

\section{Выводы}

1. Для упрощения и ускорения вычислительных процедур предложено уравнение, аппроксимирующее значения коэффициента подпора $n_{\sigma u}$, рассчитанные при помощи модифицированного метода баланса работ.

2. Точность аппроксимации расчётных данных лежит в пределах $-12 \ldots+15 \%$ что впол- не приемлемо для инженерных расчётов.

3. Результаты разработки были использованы при расчёте таблиц прокатки и проектировании нового оборудования трубопрокатных агрегатов "30-102", "350", "140" и "80".

\section{Библиографический список}

1. Целиков А. И. Влияние внешнего трения и натяжения на давление металла на валки при прокатке. Металлург. 1939. № 6. С. 3-11.

2. Лакс М. А. Расчёт потребной мощности на горячую прокатку для станов с гладкой бочкой. Технический бюллетень МПК "Центроэлектромонтаж". 1940. № 4-5. С. 38-52.

3. Целиков А. И., Гришков А. И. Теория прокатки. М. : Металлургия, 1970. 358 с.

4. Гуляев Ю. Г., Шифрин Е. И., Фролов Я. В., Ремез О. А. Интерпретация метода баланса работ при определении силовых параметров прокатки. Вісник НТУ "ХПП". 2017. № 43. C. $72-85$.

5. Романцев Б. А., Гончарук А. В., Вавилкин Н. М., Самусев С. В. Обработка металлов давлением : учебник. М.: Изд. Дом МИСиС, 2008. $960 \mathrm{c}$.

6. Гуляев Ю. Г., Максимова Э. А., Володарский М. 3., Карпов А. Г. Алгоритм статистической обработки эмпирических данных. Доклады АН УССР, серия А. 1985. № 5. С. 65-68.

7. Гуляев Ю. Г., Шифрин Е. И. Адаптация модели простой продольной прокатки для анализа силовых параметров непрерывной продольной безоправочной прокатки труб. Вісник НTУ "ХПI". 2018. № 23. С. 16-22.

8. Гуляєв Ю. Г., Николаєнко Ю. М. Технологічні процеси обробки металів тиском. Частина IX : навч. посібник. Дніпро : НМетАУ, 2019. $52 \mathrm{c}$.

9. Целиков А. И. Прокатные станы. М.: Металлургиздат, 1946. 560 с.

10. Gulyaev Yu., Garmashev D., Koriaka N. Determination of energy and force parameters of rolling with work (energy) balance method. ITA Tube Journal. 2015. No. 4. P. 56-60.

\section{References}

1. Tselikov, A. I. (1939). Vliyaniye vnechnego treniya i natyazeniya na davleniye metalla na valki pri prokatke. Metallurg, (6), 3-11

2. Lax, M. A. (1940). Raschet potrebnoy moshnosti na goryachuyu prokatku dlya stanov $\mathrm{s}$ gladkoy bochkoy. Tehnicheskiy byulleten MPK "Centroelectromontazh", (4-5), 38-52 
3. Tselikov, A. I., \& Grishkov, A. I. (1970). Teoriya prokatki. Moskva: Metallurgiya

4. Gulyaev, Yu. G., Shifrin, E. I., Frolov, Y. V., \& Remez, O. A. (2017). Interpretatsiya metoda balansa rabot pri opredelenii silovih parametrov prokatki. Visnik NTU "KhPI", (43), 72- 85

5. Romantsev, B. A., Goncharuk, A. V., Vavilkin, N. M., \& Samusev, S. V. (2008). Obrabotka metallov davleniem. Moskva : Izd. dom MISiS

6. Gulyaev, Yu. G., Maksimova, E. A., Volodarsky, M. Z., \& Karpov, A. G. (1985). Algoritm statisticheskoy obrabotki empiricheskih dannih. Doklady AN USSR, seriya A (5), 65-68

7. Gulyaev, Yu. G., \& Shifrin, E. I. (2018).
Adaptaciya modeli prostoy prodol'noy prokatki dlya analyza silovih parametrov neprerivnoy prodol'noy bezopravochnoy prokatki trub. Visnik NTU "KhPI, (23), 16-22

8. Gulyaev, Y. G., \& Nikolaenko, Y. M. (2019). Tehnologichni procesi obrobki metaliv tyskom. Chastina IX: navch. posibnik. Dnipro: NMetAU

9. Tselikov, A. I. (1946). Prokatniye stani. Moskva: Metallurgizdat

10. Gulyaev, Yu., Garmashev, D., \& Koriaka, N. (2015). Determination of energy and force parameters of rolling with work (energy) balance method. ITA Tube Journal, (4), 56-60

Шифрін Євген Ісайович, доктор технічних наук, професор кафедри обробки металів тиском, Національна металургійна академія України (Дніпро, Україна). ORCID: 0000-0002-0270-2212. E-mail: shifrinei48@gmail.com

Балакін Валерій Федорович, професор, доктор технічних наук, завідувач кафедри технологічного проектування, Національна металургійна академія України (Дніпро, Україна). ORCID: 00000003-0876-7516. E-mail: balakinvf@gmail.com

Квітка Наталія Юріївна, здобувачка кафедри технологічного проектування, Національна металургійна академія України (Дніпро, Україна). ORCID: 0000-0002-8053-3952. E-mail: nataliya.kvitka@gmail.com

\section{АПРОКСИМУЮЧЕ РІВНЯННЯ ДЛЯ ВИЗНАЧЕННЯ КОЕФІЦІЕНТУ ПІДПОРУ ПРИ ПОЗДОВЖНІЙ ПРОКАТЦІ ШТАБИ}

Мета. Запропонувати рівняння, що апроксимує розрахункові значення коефіцієнта підпору з достатньою для інженерних розрахунків точністю i, тим самим, спростити визначення силових параметрів технологічних процесів прокатки.

Методика. Використовували наступну послідовність створення апроксимуючого рівняння: спочатку вибирається базове рівняння, характер зміни якого при зміні змінних параметрів якісно відповідає характеру зміни розрахункової залежності; потім базове рівняння доповнюється функціями змінних параметрів, доцільність використання яких в апроксимуючому рівнянні обгрунтовується на підставі зіставлення вихідних розрахункових даних і результатів розрахунку відповідно апроксимуючої залежності.

Результати. Отримано залежність, що апроксимує розрахункові значення коефіцієнта підпору в межах реальних значень коефіцієнта тертя, відносного обтиску і відносного радіуса валків. Наведено графіки розрахункових та апроксимованих значень коефіцієнта підпору. Розбіжність між вихідними та апроксимованими значеннями коефіцієнта підпору не перевищує $-12 \ldots+15 \%$.

Наукова новизна. Вперше отримано апроксимуюче рівняння для розрахунку коефіцієнта підпору у всьому діапазоні реальних значень коефіцієнта тертя, відносного обтиску та відносного радіуса валків.

Практична цінність. Використання розробленої апроксимуючої залежності дозволило виконати розрахунки силових параметрів деформації в станах трубопрокатних агрегатів "30102", "350", "140" і "80". Ці дані були використані при розробці таблиць прокатки та проектуванні нового обладнання.

Ключові слова: прокатка смуги, коефіцієнт підпору, апроксимуюче рівняння. 
Yevhen Shifrin, Doctor of Technical Sciences, Professor of the Department of Metal Forming, National Metallurgical Academy of Ukraine (Dnipro, Ukraine). ORCID: 0000-0002-0270-2212. Email: shifrinei48@gmail.com

Valeriy Balakin, Professor, Doctor of Technical Sciences, Head of the Department of Technological Design, National Metallurgical Academy of Ukraine (Dnipro, Ukraine). ORCID: 0000-0003-0876-7516. Email: balakinvf@gmail.com

Natalia Kvitka, Candidate of the Department of Technological Design, National Metallurgical Academy of Ukraine (Dnipro, Ukraine). ORCID: 0000-0002-8053-3952. E-mail: nataliya.kvitka@gmail.com

\section{APPROXIMATING EQUATION FOR THE DETERMINATION OF SUPPORT COEFFICIENT FOR LONGITUDINAL ROLLING}

Purpose. To propose an equation that approximates the calculated values of the support coefficient with sufficient accuracy for engineering calculations and, thereby, simplify the determination of power parameters of the technological rolling processes.

Methodology. The following sequence of creating the approximating equation was used: firstly, the basic equation is being selected, the nature of the change of which when changing the variable parameters qualitatively corresponds to the nature of the change in the calculated dependence; then the basic equation is supplemented by functions of variable parameters, the feasibility of using which in the approximating equation is justified on the basis of a comparison of the initial calculated data and the calculated results for the approximating dependence.

Results. A dependence is obtained that approximates the calculated values of the support coefficient within the real values of the coefficient of friction, relative compression, and relative radius of the rolls. The graphs of the calculated and approximated values of the support coefficient are given. The discrepancy between the initial and approximated values of the support coefficient does not exceed $-12 \ldots+15 \%$.

Originality. For the first time, an approximating equation is obtained for calculating the support coefficient over the entire range of real values of the coefficient of friction, relative compression, and relative radius of the rolls.

Practical implications. The use of the proposed approximating equation made it possible to calculate the force parameters of the deformation in the mills of tube rolling units "30-102", "350", "140", and "80". These data were used in the development of rolling tables and the design of new equipment.

Key words: strip rolling, support coefficient, approximating equation.

Шифрин Евгений Исаевич, доктор технических наук, профессор кафедры обработки металлов давлением, Национальная металлургическая академия Украины (Днепр, Украина). ORCID: 00000002-0270-2212. E-mail: shifrinei48@gmail.com

Балакин Валерий Федорович, профессор, доктор технических наук, заведующий кафедрой технологического проектирования, Национальная металлургическая академия Украины (Днепр, Украина). ORCID: 0000-0003-0876-7516. E-mail: balakinvf@gmail.com

Квитка Наталья Юрьевна, соискатель кафедры технологического проектирования, Национальная металлургическая академия Украины (Днепр, Украина). ORCID: 0000-0002-8053-3952. E-mail: nataliya.kvitka@gmail.com

\section{АППРОКСИМИРУЮЩЕЕ УРАВНЕНИЕ ДЛЯ ОПРЕДЕЛЕНИЯ КОЭФФИЦИЕНТА ПОДПОРА ПРИ ПРОДОЛЬНОЙ ПРОКАТКЕ ПОЛОСЫ}

Цель. Предложить уравнение, аппроксимирующее расчётные значения коэффициента подпора с достаточной для инженерных расчётов точностью и, тем самым, упростить определение силовых параметров технологических процессов прокатки.

Методика. Использовали следующую последовательность создания аппроксимирующего уравнения: вначале выбирается базовое уравнение, характер изменения которого при изменении варьируемых параметров качественно соответствует характеру изменения расчётной зависимости; затем 
базовое уравнение дополняется функциями варьируемых параметров, целесообразность использования которых в аппроксимирующем уравнении обосновывается на основании сопоставления исходных расчётных данных и результатов расчёта по аппроксимирующей зависимости.

Результаты. Получена зависимость, аппроксимирующая расчётные значения коэффициента подпора в пределах реальных значений коэффициента трения, относительного обжатия и относительного радиуса валков. Приведены графики расчётных и аппроксимированных значений коэффициента подпора. Расхождение между исходными и аппроксимированными значениями коэффициента подпора не превышает $-12 \ldots+15 \%$.

Научная новизна. Впервые получено аппроксимирующее уравнение для расчёта коэффициента подпора во всём диапазоне реальных значений коэффициента трения, относительного обжатия и относительного радиуса валков.

Практическая ценность. Использование разработанной аппроксимирующей зависимости позволило выполнить расчёты силовых параметров деформации в станах трубопрокатных агрегатов "30-102", "350", "140" и "80". Эти данные были использованы при разработке таблиц прокатки и проектировании нового оборудования.

Ключевые слова: прокатка полосы, коэффициент подпора, аппроксимирующее уравнение.

Рукопис надійшов 19.03.2020 